\title{
Enseñanza de la geometría con el software GeoGebra en estudiantes secundarios de una institución educativa en Lima
}

\section{Teaching of Geometry with GeoGebra Software in High School Students of an Educational Institution in Lima}

\author{
Luis Diaz-Nunja Universidad San Ignacio de Loyola, Lima, Perú \\ ORCID: https://orcid.org/0000-0003-4696-9880
}
Jorge Rodríguez-Sosa* (iD Universidad San Ignacio de Loyola, Lima, Perú ORCID: https://orcid.org/0000-0002-8440-4891

Susana K. Lingán (D) Universidad San Ignacio de Loyola, Lima, Perú ORCID: https://orcid.org/0000-0003-4587-7853

Recibido 14-07-18 Revisado 10-08-18 Aprobado 17-10-17 En línea 18-10-18

\begin{tabular}{lll} 
*Correspondencia & & Citar como: \\
\cline { 1 - 2 } Email: jrodriguezs@usil.edu.pe & & $\begin{array}{l}\text { Diaz-Nunja, L., Rodríguez-Sosa, J., \& Lingán, } \\
\text { S.K. (2018). Enseñanza de la geometría con el }\end{array}$ \\
& software GeoGebra en estudiantes secundarios \\
& de una institución educativa en Lima. Propósitos \\
& $\begin{array}{l}y \text { Representaciones, 6(2), 217-251. Doi: http:// } \\
\text { dx.doi.org/10.20511/pyr2018.v6n2.251 }\end{array}$
\end{tabular}

(C) Universidad San Ignacio de Loyola, Vicerrectorado de Investigación, 2018

(cc) BY-NC-ND Este artículo se distribuye bajo licencia CC BY-NC-ND 4.0 Internacional (http://creativecommons.org/licenses/by-nc-nd/4.0/). 


\section{Resumen}

El estudio evaluó los efectos del empleo del software GeoGebra en la enseñanza de la geometría con estudiantes secundarios en el desarrollo de sus capacidades para el razonamiento y demostración, la comunicación matemática y la resolución de problemas. El marco del estudio fue la presencia creciente de tecnologías en la enseñanza escolar de las matemáticas en jóvenes de la era digital, en un contexto donde la educación favorece la participación activa de los estudiantes en el fortalecimiento de sus propias capacidades. Los estudiantes fueron observados en dos grupos, el grupo intervenido expuesto al empleo del software GeoGebra y el grupo de comparación expuesto a una enseñanza tradicional sin el empleo del software. Ambos grupos fueron evaluados con una Prueba de Evaluación del Aprendizaje en Geometría que se aplicó en momentos antes y después de la intervención. Los resultados sugieren que el empleo del software GeoGebra tuvo efectos en el fortalecimiento de las tres capacidades, con mejoras que resultaron significativas a niveles altos. También que las puntuaciones alcanzadas en el momento después fueron favorables al grupo intervenido en las tres capacidades, con diferencias significativas a niveles moderados.

Palabras clave: Enseñanza de la geometría, software GeoGebra, tecnologías educativas, resolución de problemas. 


\section{Summary}

The study evaluated the effects of the use of GeoGebra software in the teaching of geometry with high school students in the development of their capacities for reasoning and demonstration, mathematical communication and problem-solving. The framework of the study was the increasing presence of technologies in the school teaching of mathematics in young people of the digital age, in a context where education favors the active participation of students in the strengthening of their own abilities. The students were observed in two groups, the group intervened exposed to the use of GeoGebra software and the control group exposed to traditional teaching without the use of the software. Both groups were evaluated with a Geometry Learning Assessment Test that was applied before and after the intervention. The results suggest that the use of the GeoGebra software had effects in the strengthening of the three capacities, with improvements that were significant at high levels. Also, the scores reached at the time afterwards were favorable to the group intervened in the three capacities, with significant differences at moderate levels.

Keywords: Teaching geometry, GeoGebra software, educational technologies, problem-solving. 


\section{Introducción}

El empleo de tecnologías como pizarras interactivas, tabletas, teléfonos inteligentes o software, que facilitan los aprendizajes en campos específicos, tiene una presencia creciente en la enseñanza escolar de las matemáticas en niños y jóvenes de la era digital (Korenova, 2017). Ello en línea con las tendencias actuales en la educación que favorecen unas oportunidades de aprendizaje donde la participación de los estudiantes en el fortalecimiento de sus propias capacidades es muy activa (Hernández \& Villalba, 2001). Adicionalmente, una cantidad creciente de estudios sobre los efectos del empleo de TIC en el aula las asocian con la aparición de ambientes colaborativos en los aprendizajes, mejoras en la motivación o el interés, una mayor inclinación por la indagación, o el fortalecimiento de habilidades intelectuales como el razonamiento y la resolución de problemas (De la Chica, 2010). Otros estudios (Beeland, 2002; Weaver, 2000) las asocian con mejores rendimientos y con actitudes favorables hacia las matemáticas.

Al parecer, la incorporación de software educativo en la enseñanza de la matemática -y de la geometría en particular-, en una necesidad que debe empezar a ser cubierta en el corto plazo. Sin embargo, un cambio como éste, que sólo puede darse en condiciones óptimas si en simultáneo se hacen cambios sustantivos en el currículo, es percibido también como un problema (Silva, Gros, Garrido \& Rodríguez, 2006; Hernández, 2006). Se estima que su implementación supondría re-significar y re-aprender procesos y formas de trabajo que están muy ancladas en las prácticas cotidianas de los docentes, aquellas que además conocen y dominan (Sepúlveda \& Calderón, 2007).

Por otro lado, la formación de docentes no ha sido permeable a las señales emitidas por estas necesidades de cambio ni a la evidencia que la investigación reporta. Los currículos siguen abundando en teoría pedagógica, contenidos disciplinares, didácticas o metodologías de evaluación, dejando espacios marginales para el desarrollo de las habilidades que permitirían el empleo de herramientas tecnológicas en la enseñanza (Silva, Gros, Garrido \& Rodríguez, 2006). Adicionalmente, las escasas oportunidades de formación 
tecnológica para docentes en servicio se ofrecen en forma de talleres acotados que básicamente se enfocan en brindar información general sobre los software educativos, más la transferencia de algunas de las habilidades básicas necesarias para su manejo, sin abordar la cuestión esencial de cómo integrarlos en la enseñanza (Parsad, Lewis \& Farris, 2001). Así la formación tecnológica no concluye, por lo general es un proceso trunco que lleva a que el empleo de software en las aulas sea muy limitado o simplemente no ocurra.

Tomando en cuenta lo expuesto, nos planteamos como objetivo establecer los efectos que tuvo un programa de enseñanza de la geometría que empleó el software GeogGebra en los aprendizajes de geometría de estudiantes secundarios expresados en tres capacidades: razonamiento y demostración, comunicación matemática y resolución de problemas. La pregunta a la que se tuvo que dar respuesta fue: ¿Cuáles son los efectos de una enseñanza que empleó el software GeoGebra en el aprendizaje de la geometría en estudiantes de 4to año de secundaria de una institución educativa privada en Lima? Las tres hipótesis sustantivas que guiaron la búsqueda de esta respuesta partieron del supuesto que el empleo del software, como parte de un programa mayor de aprendizaje de la geometría, mejoraría de manera significativa las capacidades mencionadas en los estudiantes. Una hipótesis adicional sostuvo que esas mejoras serían mayores a las que se pudiese alcanzar con cualquier otra intervención.

\section{Software educativo GeoGebra.}

El software multi-plataforma GeoGebra (Hohenwarter \& Preiner, 2007), combina la facilidad de uso de otros software de geometría dinámica con las versátiles posibilidades del software algebraico. La idea básica de GeoGebra es unir geometría, álgebra y cálculo, que otros paquetes abordan por separado, en un solo paquete que se puede utilizar para la enseñanza de la geometría desde el nivel elemental hasta la formación universitaria. La enseñanza de la geometría escolar desde una perspectiva dinámica es un campo relativamente nuevo en la docencia, pero con una presencia cada vez 
más frecuente y relevante (Ferreira, et al, 2009; Duval, 2000). Por ello, el National Council of Teachers of Mathematics (NCTM) promueve el uso de software de geometría dinámica en las aulas, entre ellos GeoGebra.

Según Sánchez (2003), GeoGebra posee un conjunto de atributos (tabla 1) que resultan especialmente adecuados si se busca fortalecer capacidades matemáticas en los estudiantes.

\section{Tabla 1.}

Atributos y características de GeoGebra.

\begin{tabular}{ll}
\hline \multicolumn{1}{c}{ Atributos } & \multicolumn{1}{c}{ Características } \\
\hline Constructividad & $\begin{array}{l}\text { Posibilidad de construir nuevos escenarios a partir de la combinación de } \\
\text { objetos en espacio y tiempo. Concepto estrechamente vinculado al modelo } \\
\text { constructivista de aprendizaje. }\end{array}$ \\
Navegabilidad & $\begin{array}{l}\text { Posibilidad de explorar de manera libre y flexible, a diferencia de otros } \\
\text { paquetes que emplean rutas fijas, lineales y secuenciales. }\end{array}$ \\
Interactividad & $\begin{array}{l}\text { Sistema que provee al usuario retro-alimentación en tiempo real, además de } \\
\text { adaptar o modificar dinámicamente su comportamiento en función de los } \\
\text { eventos e información recibida. }\end{array}$ \\
Calidad del contenido & $\begin{array}{l}\text { Fiabilidad, relevancia, organización y accesibilidad de la información que } \\
\text { contiene el software, que adicionalmente puede ser adaptada a diversos tipos } \\
\text { de audiencias. }\end{array}$ \\
Interfaz & $\begin{array}{l}\text { Pantalla con que el aprendiz interactúa, que captura la atención del aprendiz, } \\
\text { guía sus acciones y refleja el estado del sistema. }\end{array}$ \\
\hline
\end{tabular}

En el campo específico de la enseñanza de la geometría, los estudios sobre intervenciones que emplearon el software GoeGebra con docentes previamente calificados en su manejo, reportan que esos mismos docentes percibieron que el paquete facilitaba alcanzar ambientes de aprendizaje interactivos (Ferreira, et al, 2009), o aprendizajes basados en procesos colaborativos (García, 2011). Otros estudios que se hicieron en el marco de la capacitación misma de docentes en el empleo de GeoGebra reportan que los docentes tuvieron opiniones favorables a su empleo en la enseñanza, si se tiene como base una capacitación adecuada (Tatar \& Yilmaz, 2016; Bulut \& Bulut, 2011). Asimismo, estudios hechos con estudiantes hallaron mejoras en dimensiones como la velocidad en realizar actividades o hallar respuestas, la comprensión de conceptos, el enfoque en determinados temas o la motivación por profundizar en ellos (Mendes, et al., 2014). 


\section{Aprendizaje de la geometría.}

La geometría es considerada como un campo reflexivo que permite resolver problemas de diversa índole y comprender un mundo que ofrece una amplia gama de formas, sea en escenarios naturales o artificiales (Gamboa \& Ballestero, 2009). Es también un soporte estratégico en la formación profesional en cualquier campo, dada su aplicación en diversos contextos y escenarios, así como el rol que puede jugar en el fortalecimiento del razonamiento lógico (Báez \& Iglesias, 2007).

De acuerdo con Duval (1998), el aprendizaje de la geometría involucra, como mínimo, realizar tres actividades cognitivas: la construcción, que alude al diseño de configuraciones mediado por instrumentos geométricos; el razonamiento, vinculado con los procesos discursivos y de argumentación; y la visualización, que se enfoca en las representaciones espaciales. Jones (2002) va a un mayor nivel de desagregación al identificar las habilidades que la geometría contribuye a desarrollar en los estudiantes: visualizar, pensar críticamente, intuir, resolver problemas, conjeturar, razonar deductivamente y argumentar de manera lógica en procesos de prueba o demostración.

Las orientaciones del trabajo pedagógico dadas por el Ministerio de Educación del Perú (MINEDU) en el Diseño Curricular Nacional de Educación Básica Regular (2009) en el área de matemática, recogen estos elementos y los incluyen en una lógica de progresión que debe favorecer que el estudiante alcance unas competencias que cambian e incrementan su complejidad en cada ciclo. El logro de cada una de las cuatro competencias del área de matemática en el VII ciclo (3ro, 4to, y 5to grado de secundaria) tiene como requisito el desarrollo de capacidades, conocimientos y actitudes, que se trabajan en los llamados procesos transversales. La competencia de Geometría es: "Resuelve situaciones problemáticas de contexto real y matemático que implican el uso de propiedades y relaciones geométricas en construcción y movimiento, en el plano y el espacio, utilizando diversas estrategias de solución y justificando sus procedimientos y resultados" 
(MINEDU, 2014: pp, 6). Las capacidades transversales para ella son: razonamiento y demostración, comunicación matemática y resolución de problemas.

Según la Guía de Rutas de Aprendizaje (MINEDU, 2014), la capacidad de razonamiento y demostración consiste en argumentar generando ideas matemáticas, justificar y validar conclusiones, supuestos, conjeturas e hipótesis, respaldados en significados y propiedades de los números y operaciones. La capacidad de comunicación matemática consiste en expresar, en forma escrita o verbal, ideas matemáticas, el significado de los números y operaciones u otros procesos, haciendo uso de diferentes representaciones y un lenguaje matemático sencillo. La capacidad de resolución de problemas consiste en elaborar y usar estrategias, planificar, ejecutar y valorar estrategias heurísticas, procedimientos de cálculo, comparación o estimación, empleando diversos recursos. Esta última capacidad es esencial y su activación se debe dar en el marco de problemas cuya proximidad con el entorno del estudiante lo motiven a comprometerse con su solución, partiendo del supuesto que los caminos hacia la respuesta son diversos y que, por ello, el estudiante debe participar activamente en el proceso conectándose con los conocimientos previos o arriesgando nuevas propuestas, en situaciones donde la creatividad debe jugar un rol clave (MINEDU, 2007).

\section{Programa de intervención con el empleo del software GeoGebra.}

El programa de intervención estuvo basado en la enseñanza de una geometría dinámica con el empleo del software GeoGebra, que fue insertado en el curso de Geometría del 4to año de secundaria (Matemática del VII ciclo). El propósito del programa fue fortalecer las capacidades de razonamiento y demostración, comunicación matemática y resolución de problemas, a la vez que evaluar ese proceso. Se trabajó con un enfoque por competencias y con didácticas que enfatizaron en la problematización. Ambas orientaciones pedagógicas son parte de las recomendaciones dadas por el MINEDU como parte del Diseño Curricular Nacional de Educación Básica Regular (2009) 
en el área de matemática, y aparecen explícitas como parte del currículo secundario de la institución educativa en la que se ejecutó la intervención. Por ello, la única diferencia entre la intervención y el programa regular fue el empleo del software GeoGebra.

El programa de intervención estuvo compuesto de 11 sesiones de tres horas cada una, una hora para la presentación de los conceptos y principios geométricos necesarios, las maneras de abordar los problemas y los resultados esperados de ello; y dos horas para la resolución de problemas con el empleo del software. En total abarcó dos meses.

En cada una de las sesiones el empleo del software (dos horas por sesión) se hizo con grupos de dos estudiantes. En general, en las 11 sesiones se puso énfasis en el empleó de metodologías y didácticas basadas en la problematización. El docente no brindó conocimientos previamente elaborados sino el abordaje de temas o situaciones específicas, a partir de las cuales acompañaba a los estudiantes en la identificación de carencias o contradicciones, con el propósito de darles la forma de problemas. Estos problemas fueron el núcleo en torno al cual se organizó la totalidad del proceso de aprendizaje. En términos operativos el docente ofrecía uno o varios problemas que retasen a los estudiantes a desplegar sus capacidades para darles una explicación o proponer soluciones. Para ello, los estudiantes debían buscar, identificar y seleccionar aquella información que les fuera necesaria, emplearla para construir posibles soluciones a los problemas y comunicarlos empleando los parámetros reconocidos por la geometría.

Las características de GeoGebra, que es un software amigable para la comprobación o la demostración visual o numérica de teoremas y propiedades, favorecieron la problematización y la resolución de problemas. En las actividades diseñadas para cada sesión, donde la resolución de problemas se hizo desde el entorno del software, los estudiantes tuvieron la oportunidad de descubrir por sí mismos. El docente se limitó a realizar un acompañamiento efectivo mediante preguntas y sugerencias, de tal manera que sin imponer las soluciones ayudó a los estudiantes a elaborar sus planes 
de solución y a descubrir los resultados, con una forma de trabajo alineada con la pedagogía de Polya (1995).

\section{Método}

Se llevó a cabo un estudio observacional de carácter analítico orientado a la evaluación de cambios en las capacidades de razonamiento y demostración, comunicación matemática y resolución de problemas, que experimentaron estudiantes de 4to año de secundaria de una institución educativa privada de Lima, en el marco del aprendizaje de la geometría. Los estudiantes fueron observados en dos grupos. El grupo intervenido expuesto al curso de Geometría del 4to año de secundaria (Matemática del VII ciclo) con el empleo del software GeoGebra para la enseñanza de una geometría dinámica. El grupo de comparación expuesto también al curso de Geometría del 4to año de secundaria (Matemática del VII ciclo) pero con enseñanza tradicional, sin el empleo del software.

\section{Participantes.}

Los grupos estuvieron conformados por 24 estudiantes cada uno, con edades que oscilaron entre los 15 y 16 años, sin casos de extra-edad. De los 48 estudiantes el $40 \%$ fueron varones y el $60 \%$ mujeres. La asignación a los grupos se hizo sobre aulas pre-establecidas en función de los procesos de matrícula de la institución educativa, que es una asignación aleatoria. Se tuvo grupos (aulas) similares en sus promedios de desempeño, edades y proporciones de género.

\section{Instrumentos y técnicas.}

La Prueba de Evaluación del Aprendizaje en Geometría fue una elaboración ad hoc para los fines de este estudio. Se trató de un inventario compuesto 
por 10 ítems para evaluar las tres dimensiones observadas: razonamiento y demostración con tres ítems, comunicación matemática con tres ítems y resolución de problemas con cuatro ítems. La prueba empleó un sistema de calificación de $0 / 20$.

La validez basada en el contenido (representatividad y relevancia) se valoró a través del juicio de expertos, cuyo proceso arrojó valores de $V d e$ Aiken superiores a .75 para todos los ítems. La confiabilidad fue explorada en una muestra de similares características al grupo de estudiantes con el empleo del método test-retest aplicado con 60 días de intervalo. Se obtuvo coeficientes de correlación superiores a .80 para las tres dimensiones. Esa misma muestra sirvió para establecer la consistencia interna del instrumento a través del coeficiente alfa, que alcanzó valores de .822 para razonamiento y demostración, .867 para comunicación matemática y .850 para resolución de problemas.

\section{Procedimientos.}

La evaluación de las tres capacidades empleó la data obtenida de la Prueba de Evaluación del Aprendizaje en Geometría que se aplicó a los grupos intervenido y de comparación en momentos antes y después de la intervención. La aplicación en el momento antes corroboró la equivalencia del desempeño de ambos grupos en las capacidades evaluadas. Luego vino la intervención, con 11 sesiones de tres horas cada una, que se desarrolló en dos meses. Finalmente, la aplicación en el momento después estableció las diferencias en los desempeños mencionados entre ambos grupos.

El diseño metodológico empleado comprendió dos tipos de análisis: comparación de puntuaciones por momentos al interior de cada grupo 
(análisis intra-grupal) y por momentos entre los dos grupos (análisis intergrupal). Debido al tamaño muestral y las características del muestreo, para el análisis intra-grupal se empleó la prueba $T$ de Wilcoxon, mientras que para el análisis inter-grupal se utilizó la prueba $U$ de Mann Whitney, para luego calcular la magnitud del efecto en la forma de correlación biserial $\left(\mathrm{r}_{\mathrm{b}}\right)$ (Fritz, Morris \& Richler, 2012). Para considerar la diferencia entre grupos, se tomó en cuenta la magnitud del $\mathrm{r}_{\mathrm{b}}:<.10$ como insignificante; entre .10 y .30 , bajo, entre .30 y .50 , moderado; y mayor que .50 , alto.

\section{Resultados}

\section{Tabla 2.}

Diferencias de grupos relacionados en las capacidades geométricas evaluadas

\begin{tabular}{llcccccccc}
\hline \multirow{2}{*}{ Dimensión } & & \multicolumn{2}{c}{ Pre test } & \multicolumn{2}{c}{ Post test } & \multirow{2}{*}{$\mathrm{Z}$} & $\mathrm{p}$ & $\mathrm{r}_{\mathrm{b}}$ \\
\cline { 3 - 6 } & & $\mathrm{M}$ & $\mathrm{DE}$ & $\mathrm{M}$ & $\mathrm{DE}$ & & & \\
\hline Comunicación & G. Experimental & 2.41 & .94 & 4.27 & 1.26 & -3.89 & $.000^{* *}$ & .56 \\
matemática & G. Control & 2.45 & 1.33 & 3.45 & 1.19 & -3.07 & $.002^{* *}$ & .44 \\
\multirow{2}{*}{$\begin{array}{l}\text { Razonamiento y } \\
\text { demostración }\end{array}$} & G. Experimental & 1.58 & .77 & 2.62 & .78 & -3.37 & $.001^{* *}$ & .49 \\
& G. Control & 1.66 & .88 & 2.14 & .71 & -2.33 & $.019^{*}$ & .34 \\
$\begin{array}{l}\text { Resolución de } \\
\text { problemas }\end{array}$ & G. Experimental & 2.66 & 1.07 & 4.89 & 1.75 & -3.64 & $.000^{* *}$ & .53 \\
& G. Control & 2.58 & .90 & 3.77 & .84 & -3.67 & $.000^{* *}$ & .53 \\
\hline
\end{tabular}

$* \mathrm{p}<.05$

$* * \mathrm{p}<.01$

A nivel intra-grupal (tabla 1), en ambos grupos la evaluación de las capacidades de razonamiento y demostración, comunicación matemática y resolución de problemas, resultó siempre favorable al momento después con diferencias estadísticamente significativas. En el análisis complementario para establecer la significancia práctica de las diferencias, sólo el grupo intervenido alcanzó niveles altos $\left(\mathrm{r}_{\mathrm{b}}>.50\right)$, mientras el grupo de comparación presentó niveles moderados $\left(\mathrm{r}_{\mathrm{b}}>.30\right)$. 


\section{Tabla 3.}

Diferencias de grupos independientes en las capacidades geométricas evaluadas

\begin{tabular}{|c|c|c|c|c|c|c|c|c|}
\hline \multirow{2}{*}{ Dimensión } & & \multicolumn{2}{|c|}{ Grupo experimental } & \multicolumn{2}{|c|}{ Grupo control } & \multirow{2}{*}{$\mathrm{U}$} & \multirow{2}{*}{$\mathrm{p}$} & \multirow{2}{*}{$r_{b}$} \\
\hline & & $\mathrm{M}$ & DE & $\mathrm{M}$ & $\mathrm{DE}$ & & & \\
\hline \multirow{2}{*}{$\begin{array}{l}\text { Comunicación } \\
\text { matemática }\end{array}$} & Pre test & 2.41 & .94 & 2.45 & 1.33 & 264.50 & .623 & .07 \\
\hline & Post test & 4.27 & 1.26 & 3.45 & 1.19 & 179.50 & $.024 *$ & .33 \\
\hline \multirow{2}{*}{$\begin{array}{l}\text { Razonamiento } \\
\text { y demostración }\end{array}$} & Pre test & 1.58 & .77 & 1.66 & .88 & 265.50 & .636 & .06 \\
\hline & Post test & 2.62 & .78 & 2.14 & .71 & 190.00 & $.039 *$ & .30 \\
\hline \multirow{2}{*}{$\begin{array}{l}\text { Resolución de } \\
\text { problemas }\end{array}$} & Pre test & 2.66 & 1.07 & 2.58 & .90 & 262.00 & .586 & .07 \\
\hline & Post test & 4.89 & 1.75 & 3.77 & .84 & 173.00 & $.016^{*}$ & .35 \\
\hline
\end{tabular}

$* \mathrm{p}<.05$

A nivel inter-grupal (tabla 2), el momento antes arrojó puntuaciones similares en ambos grupos para las tres capacidades. En el momento después se halló diferencias estadísticamente significativas entre los grupos $(\mathrm{p}<.05)$ en las tres capacidades, siempre favorables al grupo intervenido. Además, se estableció que estas diferencias alcanzan sólo un nivel moderado en todos los casos $\left(\mathrm{r}_{\mathrm{b}}>.30\right)$.

\section{Discusión}

Los resultados nos permitieron alcanzar lo previsto en el objetivo, en el sentido que el empleo de un software como GeoGebra en el marco de un curso de geometría en secundaria tuvo efectos importantes en los aprendizajes de los estudiantes involucrados en el estudio, en lo referido al fortalecimiento de sus capacidades de razonamiento y demostración, comunicación matemática y resolución de problemas. Adicionalmente, sugieren efectos colaterales como el facilitar el trabajo en grupos, favorecer los procesos de colaboración en el aprendizaje y recoger opiniones favorables sobre el empleo del software en el docente.

Las puntuaciones obtenidas en la aplicación de la Prueba de Evaluación del Aprendizaje en Geometría fueron superiores en ambos grupos en el 
momento después en las tres capacidades evaluadas, demostrando que cualquier intervención tiene efectos positivos. Sin embargo, estos resultados mostraron ciertas tendencias. Entre los momentos antes y después el grupo intervenido alcanzó diferencias estadísticamente significativas a niveles altos (rbis > .50), mientras el grupo de comparación sólo alcanzó niveles moderados (rbis $>.30)$. En el momento después, luego de la paridad observada en el momento antes, el grupo intervenido alcanzó puntuaciones superiores a las del grupo de comparación, con diferencias significativas a niveles moderados (rbis > .30). La intervención con el empleo del software GeoGebra demostró que produce cambios más significativos que la enseñanza tradicional.

Estos resultados coinciden con los reportados por otros estudios sobre los efectos del empleo de TIC o, en específico, del software GeoGebra en el aula. De la Chica (2010), encontró que el empleo de TIC lleva a que los estudiantes alcancen mejoras en habilidades intelectuales como el razonamiento y la resolución de problemas. Mendes, et al., (2014), halló que el uso de GeoGebra tiene incidencia en que los estudiantes alcancen mejoras en la velocidad para realizar actividades o para llegar a respuestas, así como en la comprensión de conceptos.

Es lógico deducir que efectos como los reportados se produjeron porque se contó con ciertas condiciones favorables para que ello ocurra. Una de estas condiciones tuvo que ver con el hecho de que el docente a cargo de la intervención tuvo las competencias necesarias para integrar el software GoeGebra como parte de su enseñanza, acercando su empleo real al potencial del software mismo. Esta es una condición indispensable resaltada en un estudio previo por Parsad, Lewis \& Farris (2001), quienes sostienen que las habilidades tecnológicas de los docentes son claves para integrar las TIC en un marco de buena enseñanza. En ese sentido, una capacitación tecnológica integral resulta estratégica para que un software como GoeGebra se torne en una herramienta productiva y, lo que es más importante, los docentes la perciban como tal (Tatar \& Yilmaz, 2016; Bulut \& Bulut, 2011). 
Otra condición favorable fue que la institución educativa donde se ejecutó la intervención, tiene como orientación pedagógica ambientes de aprendizaje donde se trabaja en grupos para el abordaje de temas o la solución de problemas. Los estudiantes están acostumbrados a estudiar de esa manera. En ese sentido, el esquema de trabajo en parejas no sólo se facilitó sino que, progresivamente, se tornó en un trabajo colaborativo donde se complementaron habilidades, con un efecto importante en cómo y cuánto se pudo realizar de las tareas asignadas. Las parejas contribuyeron decisivamente a la superación de obstáculos, o al menos a la percepción de que las dificultades fueron menos de las que se hubiesen tenido de haber realizado los trabajos en solitario. Este hallazgo es consistente con lo reportado por otros estudios que emplearon GeoGebra en el campo de las matemáticas y que hallaron ambientes colaborativos de aprendizaje (García, 2011; Lavy \& Leron, 2004) y de interacción (Ferreira, et al, 2009) emergentes.

En opinión del docente a cargo de la enseñanza en los grupos comparados, GeoGebra resultó ser un programa de fácil manejo para los estudiantes, que requirió de poco tiempo para que se familiarizasen con los recursos que ofrecía. Sostuvo también que el empleo del software facilitó que los estudiantes entendiesen conceptos difíciles, ayudando a desaparecer algunos obstáculos de la enseñanza en este campo, opinión que es consistente con las reportadas en otros estudios (Mendes, et al., 2014; Ferreira, et al., 2009).

Como conclusiones podemos sostener que las cuatro hipótesis formuladas fueron validadas. El análisis intra-grupo reportó que en todos los casos (capacidades de razonamiento y demostración, comunicación matemática y resolución de problemas) las puntuaciones fueron superiores en el momento después, con diferencias que fueron significativas a niveles altos $\left(\mathrm{r}_{\mathrm{b}}>.50\right)$, E1 análisis inter-grupos reportó que las puntuaciones alcanzadas en el momento después fueron favorables en todos los casos al grupo intervenido, con diferencias que resultaron significativas a niveles moderados $\left(\mathrm{r}_{\mathrm{b}}>.30\right)$. 
Finalmente, la experiencia tenida nos lleva a sostener que la investigación sobre la propia práctica docente puede tener importantes implicancias para la enseñanza, proporcionando evidencia favorable para el empleo de estrategias o herramientas, o simplemente proporcionando ideas, que puedan brindar a los docentes la oportunidad de utilizarlas en procesos de mejora. A diferencia de una investigación con propósitos fundamentalmente cognitivos, que busca la producción de un conocimiento generalizable y, por ello, difícilmente aplicable a las condiciones particulares de la práctica cotidiana de un docente concreto, la investigación aplicada genera aprendizajes desde situaciones comunes, que resultan relevantes y fáciles de ser abordados.

\section{Referencias}

Báez, R., \& Iglesias, M. (2007). Principios didácticos a seguir en el proceso de enseñanza y aprendizaje de la geometría en la UPEL “El Mácaro". Enseñanza de la Matemática, 12(16), 67-87.

Beeland, J. W. D. (2002). Student engagement, visual learning and technology: Can interactive white-boards help? Action Research Exchange, 1(1). Recuperado de: http://citeseerx.ist.psu.edu/viewdoc/download?doi=10.1.1.1 $35.3542 \&$ rep $=$ rep $1 \&$ type $=$ pdf

Bulut, M., \& Bulut, N. (2011). Pre service teachers' usage of dynamic mathematics software. TOJET: The Turkish Online Journal of Educational Technology, 10(4), 294-299.

De la Chica, J. (2010). Metodologías activas y aprendizaje por descubrimiento. Las TIC y la educación. Almería: Tutorial Formación, S.L.L.

Duval, R. (2000). Basic issues for research in mathematics education. En T. Nakahara y M. Koyama (Eds.), Procedings of the 24th PME international conference, 2 (pp. 55-69). Ciudad: Hiroshima, Japón: PME.

Ferreira, A., Guerra Cardoso, H., Santos Macedo, E., Breviario, D. \& ArnholdtSchmitt, B. (2009). Trabajo conjetural con el uso de GeoGebra (Ed.), Memorias del sexto Congreso Internacional de Enseñanza de la Matemática Asistida por Computadora (CIEMAC 6). Costa Rica, Cartago. 
Fritz, C. O., Morris, P. E. \& Richler, J. J. (2012). Effect size estimates: current use, calculations, and interpretation. Journal of Experimental Psychology: General, 141(1), 2 - 18. Doi: https://doi.org/10.1037/a0024338

Gamboa, R., \& Ballestero, A. (2009). Algunas reflexiones sobre la didáctica de la geometría. Cuadernos de Investigación y Formación en Educación Matemática, 4(5), 113- 136.

García, M. P. (2011). Evolución de actitudes y competencias matemáticas en estudiantes de secundaria al introducir geogebra en el aula (Tesis de Doctorado). Universidad de Almería, España.

Hernández, V. \& Villalba, M. (2001). Perspectivas en la Enseñanza de la geometría para el siglo XXI. Documento de discusión para estudio ICMI. PMME-UNISON. Traducción del documento original. Recuperado de: http://www.euclides.org/menu/articles/article2.htm

Hernández, O. (2006). ¿Cambiarán las computadoras la forma de enseñar la geometría? Recuperado de: http://biblo.una.edu.ve/docu.7/bases/marc/ texto/m2451b.pdf

Hohenwarter, M. \& Preiner, J. (2007) Dynamic mathematics with GeoGebra. Journal of online mathemtatics and its applications, art. $\mathrm{N}^{\circ} 1448$, International GeoGebra Institute, https://www.geogebra.org/

Jones, K. (2002). Issues in the Teaching and Learning of Geometry. En L. Haggarty (Ed.), Aspects of Teaching Secondary Mathematics: perspectives on practice, pp. 121-139. London: Routledge Falmer.

Korenova, L. (2017). GeoGebra in teaching of primary school mathematics. International Journal of Technology in Mathematics Education, 24(3). 155160. Doi:

Lavy, I., \& Leron, U. (2004). The Emergence of Mathematical Collaboration in an interactive Computer Environment. International Journal of Computers for Mathematical Learning. 9(1), 1-23. https://doi.org/10.1023/ B:IJCO.0000038244.16252.45

Mendes, T., Nascimento, G., Coelho Neto, J., Luccas, S., Pereira, R., \& Bernardelli, M., (2014). The use of software Geogebra as an instrument of learning for the Mathematics. Revista Espacios, 35(6), 2-6. Recuperado de: http://www. revistaespacios.com/a14v35n06/14350602.html

Ministerio de Educación - MINEDU. (2009). Diseño Curricular Nacional de Educación Básica Regular. Lima: MINEDU. 
Ministerio de Educación - MINEDU. (2014). Rutas de aprendizaje. Hacer uso de saberes matemáticos para afrontar desafios diversos. Área curricular, Matemática, VII ciclo. Lima: MINEDU.

Ministerio de Educación - MINEDU. (2007). Aspectos metodológicos en el aprendizaje de los sistemas de números naturales, enteros, racionales y reales en secundaria. Fecha de consulta: 24/12/2014. <http://sistemas02. minedu.gob.pe/archivosdes/fasc_mat/04_mat_d_s2_f1.pdf $>$

Ministerio de Educación - MINEDU. (2007). Guía de Aprendizaje de la Matemática y el Desarrollo de Capacidades. Fascículo 2. Lima: MINEDU.

Parsad, B., Lewis, L., \& Farris, E. (2001). Teacher Preparation and Professional Development. ERIC. Search Education Resources.

Polya, G. (1995). Cómo plantear y resolver problemas. México: Trillas.

Sánchez, E. (2003). La demostración en geometría y los procesos de reconfiguración: una experiencia en un ambiente de geometría dinámica. Educación Matemática, 15 (2), 27-53.

Sepúlveda, M.P., \& Calderón, I. (2007). Las TIC y los procesos de enseñanzaaprendizaje: la supremacía de las programaciones, los modelos de enseñanza y las calificaciones ante las demandas de la sociedad del conocimiento. Revista Iberoamericana de Educación. 44(5), 1-13. Recuperado de: https:// rieoei.org/RIE/article/view/2217

Silva, J., Gros, B., Garrido, J. M., \& Rodríguez, J. (2006). Estándares en tecnologías de la información y la comunicación para la formación inicial docente: situación actual y el caso chileno. Revista Iberoamericana De Educación, 38(3), 1-17. Recuperado de: https://rieoei.org/RIE/article/ view/2658

Tatar, E., \& Yilmaz, Z. (2016) Conceptual Understanding of Definite Integral with GeoGebra. Computers in the schools. Interdisciplinary Journal of Practice, Theory and Applied Research, 33(2), 120-132. Doi: https://doi.org/10.1080/ 07380569.2016.1177480

Weaver, G. (2000). An examination of the national educational longitudinal study (NELS: 88) database to probe the correlation between computer use in school and improvement in test scores. Journal of Science Education and Technology, 9, 121-133. Doi: https://doi.org/10.1023/A:1009457603800 